\title{
FreqüÊNCIA DE SINTOMAS DIGESTIVOS EM PACIENTES brasileiros com Diabetes Mellitus
}

\author{
L.E. de A. Troncon*, R.P. Lopes, M.N. Simão, M. lquegami, L. Rosa-e-Silva, \\ M.A. Nobre-e-Souza, M.C. Foss \\ TrabalhorealizadonoDepartamento deClínicaMédica, FaculdadedeMedicinade RibeirãoPreto \\ daUniversidadedeSãoPaulo, RibeirãoPreto,SP.
}

Resumo - Sintomas sugestivos do comprometimento do tubo digestivo são comuns em pacientes diabéticos, mas estudos comparando as freqüências destes sintomas com as da população geral são escassos e não existem trabalhos dessa natureza com diabéticos brasileiros.

ОвJetivo. Determinar as freqüências de diferentes sintomas digestivos em amostra não selecionada de pacientes diabéticos, em comparação às encontradas em pessoas da comunidade.

MÉtodos. A ocorrência de 13 diferentes sintomas digestivos foi investigada por meio da aplicação de um questionário padronizado, especificamente estruturado, em I53 diabéticos dos tipos I e 2 e em 50 pessoas aparentemente sadias, tomadas como controles.

Resultados. A frequiência de diabéticos com pelo menos um sintoma digestivo foi significativamente superior à verificada no grupo controle $(70 \%$ vs $36 \%, p=0,01)$. Freqüências elevadas de plenitude gástrica ( $30 \%$ vs $36 \%)$, pirose ( $30 \%$ vs $34 \%)$ e de constipação (17\% vs $12 \%$ ), foram observadas tanto entre os diabéticos como nos controles. No entanto, somente a freqüência de um único sintoma, a disfagia, foi significativamente maior entre os diabéticos, em relação ao grupo controle (I3\% vs $2 \%$, $p=0,02$ ).

Conclusão. Estes resultados indicam que a freqüência de sintomas digestivos em diabéticos, apesar de elevada, não difere da verificada na população geral, exceto para a disfagia. As freqüências dos diferentes sintomas estiveram próximas das faixas relatadas em estudos do hemisfério Norte, 0 que não apóia a hipótese de que fatores inerentes ao meio afetam a ocorrência de queixas digestivas em diabéticos.

UnIteRmos: Diabetes Mellitus. Sintomas gastrointestinais. Motilidade gastrointestinal. Disfagia. Dispepsia.

\section{INTRODUÇÃO}

O Diabetes Mellitus é uma doença metabólica crônica, muito freqüente em todo o mundo, que pode afetar muitos órgãos e aparelhos'. No aparelho digestivo, em particular, é relativamente bem conhecida a ocorrência de complicações do Diabetes Mellitus, como os distúrbios da motilidade do tubo digestivo e as alterações da absorção de água e de eletrólitos ${ }^{2,3}$, bem como a litíase biliar e a esteatose hepática ${ }^{4}$. Em decorrência do comprometimento do tubo

*Correspondência:

*Prof. Dr. Luiz Ernesto deAlmeida Troncon

Hospital das Clínicas-Campus daUSP_Cep: 14048-900

RibeirãoPreto-SP-Tel(16) 633-0436-Fax(16) 633-6695 E-mail:ledatron@fmrp.usp.br digestivo, pacientes diabéticos podem apresentar quadros específicos, sendo alguns de grande relevância clínica, como a gastroparesia diabética ${ }^{4-6}$ e a diarréia do diabéti$\mathrm{co}^{2-4}$.

Sintomas bem definidos, atribuíveis ao trato gastrointestinal, são comuns em pacientes diabéticos ${ }^{2,4-12}$. A presença de pelo menos uma queixa digestiva pode ocorrer em mais de três quartos dos casos de diabetes e sintomas como disfagia, dispepsia, dor abdominal, constipação intestinal, diarréia e incontinência fecal podem, isoladamente, incidir em proporções variáveis de casos, que vão de um quinto a um terço dos diabéticos, dependendo do sintoma estudado ${ }^{2}$. No entanto, estudos mais objetivos da prevalência de sintomas digestivos em diabéticos, com análise comparativa à freqüência encontrada na população geral, não são muito numerosos ${ }^{5-11}$. Além disso, em pelo um destes estudos, feito em amostra expressivamente numerosa, a prevalência de sintomas gastrointestinais nos pacientes diabéticos não foi significativamente diferente da verificada em controles ${ }^{10}$.

É sabido que os sintomas das diversas condições mórbidas e, em especial, os relacionados ao aparelho digestivo, constituem manifestações clínicas subjetivas, que são passíveis de serem influenciadas por fatores externos, como os ligados aos aspectos culturais, à raça, à dieta e ao clima, entre outros. Assim sendo, é lícito supor que os resultados de estudos sobre prevalência de sintomas em diabéticos realizados no hemisfério Norte 
possam não ser generalizados para o Brasil, onde, ao nosso conhecimento, não existem trabalhos desta natureza.

Tendo em vista estas considerações, constituiu o objetivo deste trabalho determinar a freqüência de sintomas digestivos em uma amostra de pacientes diabéticos brasileiros, em comparação à verificada em pessoas hígidas provenientes da população geral.

\section{Métodos}

Foram investigados 153 pacientes com Diabetes Mellitus, sendo 26 do tipo I e I 27 do tipo 2, e 50 pessoas aparentemente sadias, que integraram o grupo controle. As características gerais dos dois grupos estudados são apresentadas na Tabela I. A amostra de diabéticos foi selecionada ao acaso dentre os pacientes do Ambulatório de Diabetes da Divisão de Endocrinologia e Metabologia do Departamento de Clínica Médica da FMRP-USP e representou cerca de $20 \%$ do conjunto total de pacientes acompanhados na instituição. Todos os casos preenchiam critérios diagnósticos aceitos internacionalmente ${ }^{13}$. Foram considerados como diabéticos do tipo I (DM-I), aqueles com registro objetivo de antecedentes de cetoacidose e que necessitavam do uso regular de insulina para controle da doença. Foram considerados como diabéticos do tipo 2 (DM-2), os casos que nunca apresentaram cetoacidose e eram tratados somente com dieta ou com a associação de dieta e drogas antidiabéticas orais.

Os integrantes do grupo controle foram selecionados ao acaso entre acompanhantes de pacientes atendidos em três unidades de saúde afiliadas à FMRP-USP (Hospital das Clínicas, Unidade de Emergência e Centro de Saúde-Escola). Os critérios de inclusão neste grupo foram: a) não ser portador de nenhuma doença aguda ou crônica conhecida; b) não ser portador conhecido de DM; c) não estar em acompanhamento médico regular; d) não fazer uso rotineiro de qualquer medicação.

Todos os pacientes do estudo deram seu consentimento verbal à sua inclusão na investigação, após serem devidamente informados dos seus objetivos e da sua natureza. A pesquisa foi realizada segundo recomendações éticas internacionais baseadas na Declaração de Helsinki ${ }^{14}$.

\section{Instrumento}

O instrumento principal do estudo foi um questionário estruturado e padronizado, especificamente elaborado para esta finalidade a partir de informações de trabaIhos de prevalência geral de sintomas digestivos em amostras populacionais variadas 15,16. Antes da aplicação do questionário, eram registrados dados demográficos contidos nos prontuários dos pacientes ou fornecidos pelos sujeitos do grupo controle.

O questionário continha um total de 34 itens, distribuídos em três seções (manifestações esofageanas, dispépticas e intestinais), para a caracterização de 13 sintomas. Para cada uma das manifestações presentes, era inqüirida a sua freqüência e a sua duração, além de outros dados, de acordo com o tipo de sintoma (por exemplo, tipo de alimento associado à disfagia, relação com a ingestão de alimentos para sintomas dispépticos, localização de dor ou desconforto abdominal, freqüência das evacuações e consistência das fezes). A ocorrência de cada um dos sintomas era investigada a partir de questões genéricas escritas no questionário na linguagem habitualmente empregada por pessoas leigas, que eram lidas para os pacientes, de modo a uniformizar o vocabulário e a entonação das perguntas.

$\mathrm{Na}$ análise das respostas foram adotadas as seguintes definições:

\section{I) Sintomas esofageanos}

a) disfagia - sensação de dificuldade para engolir ou sensação de parada de alimentos no percurso da boca ao estômago, logo após engolir; b) dor retroesternal - sensação muito desconfortável e dolorosa sentida no peito, atrás do esterno; c) pirose sensação de azia ou de queimação percebida no peito, atrás do esterno, ou com tendência ascendente, do estômago até a boca; d) regurgitação - sensação de volta involuntária de alimento ou de líquido do estômago até a garganta ou a boca, não precedida da sensação de enjôo;

\section{2) Sintomas dispépticos}

e) plenitude epigástrica pós-prandial - sensação de estar "cheio", "estufado", "empachado" ou "empanzinado", logo após comer, tendo ingerido volume habitual dos alimentos; f) náuseas - sensação de estar com "enjôo" ou sentir-se na iminência de vomitar; g) vômitos - eliminação repentina do conteúdo do estômago, precedida de náuseas; h) dor epigástrica - sensação muito desconfortável e dolorosa percebida no epigástrio.

\section{3) Sintomas intestinais}

i) distensão abdominal - percepção de que todo o abdome está distendido, "estufado"ou "tenso"; j) dor abdominal - sensação dolorosa, em torção, aliviada pela eliminação de gazes ou fezes; k) diarréia - eliminação de fezes de consistência líquida ou pastosa-amolecida, na freqüência de duas ou mais vezes ao dia; I) constipação intestinal eliminação, com alguma dificuldade, de fezes de consistência ressecada ou endurecida, na freqüência de uma vez a cada dois ou mais dias; m) incontinência fecal - eliminação imperceptível de fezes, de modo a sujar as roupas.

A ocorrência de cada um dos sintomas somente foi registrada quando o entrevistado respondia afirmativamente às questões respectivas, de modo a preencher os requisitos das definições e quando referia freqüência de acometimento de duas ou mais vezes por semestre e duração superior a três meses. 


\section{Análise estatística}

Para a análise da diferença de médias da variável idade foi utilizado o teste "t" de Student. Esta variável foi a seguir submetida à uma análise estratificada, utilizando-se a idade de 40 anos como ponto de corte para a definição de dois estratos. O cálculo do odds ratio ajustado foi feito pela técnica de Mantel-Haenszel. As comparações entre as proporções de diabéticos e de controles apresentando ou não sintomas, bem como as associações entre variáveis qualitativas, foram analisadas pelo teste exato de Fisher ${ }^{17}$. Em todas as situações, adotou-se como limite de significância estatística um valor de "p" igual a 0,05.

Para a realização dos cálculos estatísticos foram utilizados os programas GraphPad Instat, versão 3.0 (GraphPad Software Inc., U.S.A.) e Stata, versão 6.0 (Stata Corporation, USA).

\section{Resultados}

\section{Freqüência geral de sintomas}

A Figura I mostra que a proporção de pacientes diabéticos apresentando pelo menos um sintoma digestivo (70\%) foi significativamente superior $(p<0,0 \mathrm{l})$ à verificada entre os controles (36\%). Esta diferença persistiu mesmo com o ajuste da idade pelo odds ratio de Mantel-Haenszel, o qual mostrou um valor de 2,5I (IC 95\%, $1,19-5,28 ; p=0,0 \mid 4)$. Esta análise foi necessária, uma vez que a idade dos diabéticos (média = 53,2 anos; desvio-padrão = I5 anos), foi significativamente maior $(p>0,0001)$ que a dos controles (média = 33,I anos; desvio-padrão $=10,4$ anos), conforme mostram os dados da Tabela 1.

A Tabela 2 apresenta a freqüência dos diferentes sintomas digestivos nos dois grupos estudados. Estes dados revelam que as manifestações relacionadas às porções proximais do tubo digestivo, com a plenitude epigástrica pós-prandial e a pirose, constitu-
Tabela I - Características gerais dos grupos estudados. 0 grupo de diabéticos (DM) foi composto por pacientes do tipo I (DM I) e do tipo 2 (DM 2).

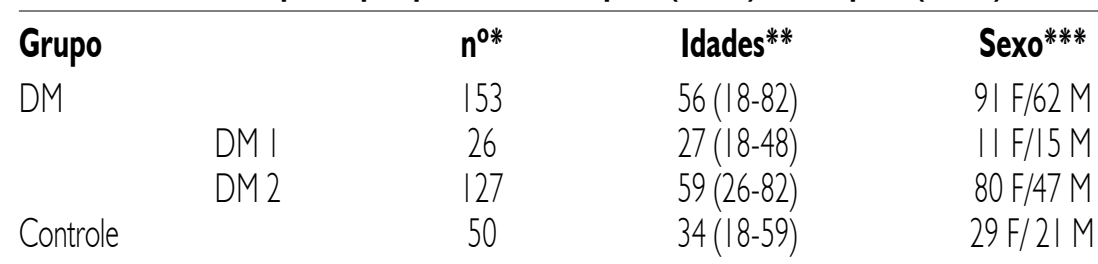

* n- número de participantes; ** - mediana e (faixa de variação), em anos; ${ }^{* * *}$ - $\mathrm{F}$ - sexo feminino, M - sexo masculino.

Figura I - Porcentagem de pacientes dos grupos de diabéticos (coluna hachurada, $n=153$ ) e de voluntários do grupo controle (coluna clara, $n=50$ ) apresentando pelo menos um sintoma digestivo à aplicação de um questionário estruturado e padronizado. A proporção de diabéticos com pelo menos um sintoma digestivo foi significativamente superior à de controles.

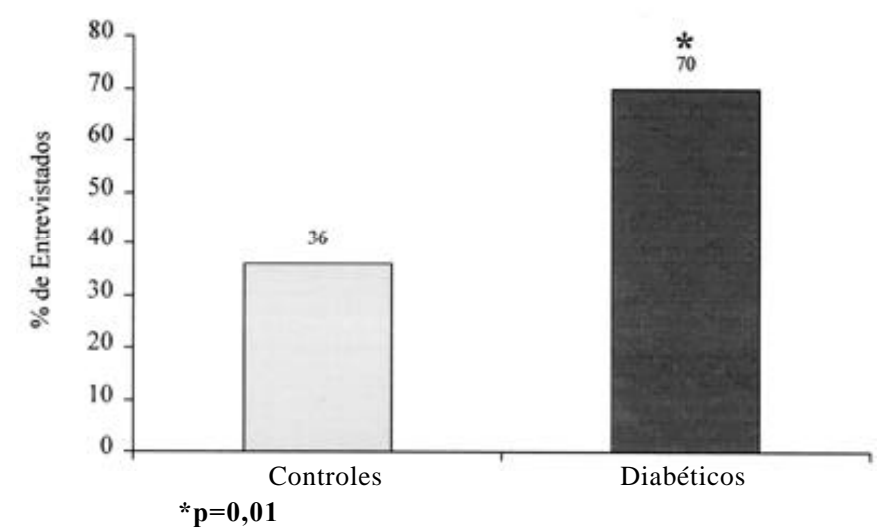

Figura 2 - Porcentagens de pacientes dos grupos de diabéticos (coluna hachurada, $n=153$ ) e de voluntários do grupo controle (coluna clara, $n=50$ ) apresentando diferentes sintomas esofageanos. Somente a freqüência de disfagia foi significativamente superior entre os diabéticos.

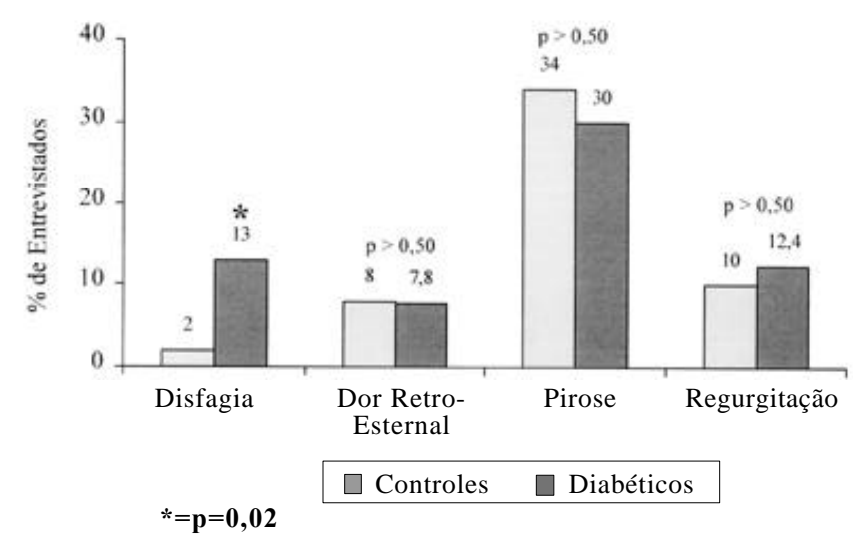


íram os sintomas mais freqüentemente registrados, tanto no grupo de diabéticos, como no grupo controle.

\section{Sintomas esofageanos}

A Figura 2 mostra as freqüências dos sintomas esofageanos, disfagia, dor retrosternal, pirose e regurgitação, nos dois grupos estudados. A freqüência de disfagia entre os diabéticos (13,0\%) foi significativamente superior $(p=0,02)$ à verificada entre os controles (2,0\%). Não houve diferenças entre os dois grupos quanto à freqüência dos demais sintomas esofageanos.

\section{Sintomas dispépticos}

A Figura 3 mostra a freqüência dos sintomas plenitude epigástrica pós-prandial, dor e/ou desconforto epigástrico, náuseas e vômitos, nos grupos de diabéticos e controle. Não houve diferença estatisticamente significativa entre os dois grupos com relação à freqüência de qualquer um destes sintomas.

\section{Sintomas intestinais}

A Figura 4 mostra a freqüência dos sintomas distensão abdominal, dor abdominal, diarréia, constipação intestinal e incontinência fecal. As proporções de diabéticos com estes sintomas foram superiores às verificadas no grupo controle. Não houve, porém diferenças estatisticamente significativas entre os dois quanto à freqüência de qualquer sintoma deste tipo.

\section{Comparação entre os subgrupos de diabéticos}

A Tabela 3 apresenta a freqüência de alguns sintomas digestivos nos dois subgrupos de pacientes diabéticos, ou seja, naqueles com diabetes do tipo I (DMI) e nos do tipo 2 (DM-2). Não houve diferenças estatisticamente significativas na comparação entre os dois subgrupos quanto às proporções dos sintomas investigados

Tabela 2 - Freqüência de sintomas digestivos nos grupos de diabéticos (DM) e de controles (C). Os dados apresentados são os números e, entre parênteses, as porcentagens de sujeitos acometidos. Apresentam-se, também, os valores de p para a análise estatística (teste de Fisher) das diferenças entre os grupos.

\begin{tabular}{|c|c|c|c|}
\hline \multirow[b]{2}{*}{ Sintomas } & \multicolumn{3}{|c|}{ GRUPOS } \\
\hline & $\mathrm{DM}(\mathrm{n}=153)$ & $C(n=50)$ & p \\
\hline - Plenitude epigástrica pós-prandial & $46(30,6)$ & $18(36,0)$ & 0,48 \\
\hline - Pirose & $45(30,0)$ & $17(34,0)$ & $>0,50$ \\
\hline - Dor abdominal & $30(19,6)$ & II $(22,0)$ & $>0,50$ \\
\hline - Distensão abdominal & $29(18,9)$ & $8(16,0)$ & $>0,50$ \\
\hline - Náuseas & $28(18,3)$ & $9(18,0)$ & $>0,50$ \\
\hline - Constipação Intestinal & $26(16,9)$ & $6(12,0)$ & $>0,50$ \\
\hline - Dor epigástrica & $24(15,6)$ & $7(\mid 4,0)$ & $>0,50$ \\
\hline - Disfagia & $20(13,0)$ & $I^{\prime}(2,0)$ & $0,02 *$ \\
\hline - Regurgitação & $19(12,4)$ & $5(10,0)$ & $>0,50$ \\
\hline - Dor retrosternal & $12(7,8)$ & $4(8,0)$ & $>0,50$ \\
\hline - Vômitos & $11(7,2)$ & $4(8,0)$ & $>0.50$ \\
\hline - Diarréia & $\|(7,2)$ & $0(0)$ & 0,19 \\
\hline - Incontinência fecal & $7(4,5)$ & $0(0)$ & 0,45 \\
\hline
\end{tabular}

Figura 3 - Porcentagens de pacientes dos grupos de diabéticos (coluna hachadura, $n=153$ ) e de voluntários do grupo controle (coluna clara, $n=50$ ) apresentando diferentes sintomas dispépticos. Não houve diferenças significativas entre os dois grupos quanto à freqüência de nenhum destes sintomas.

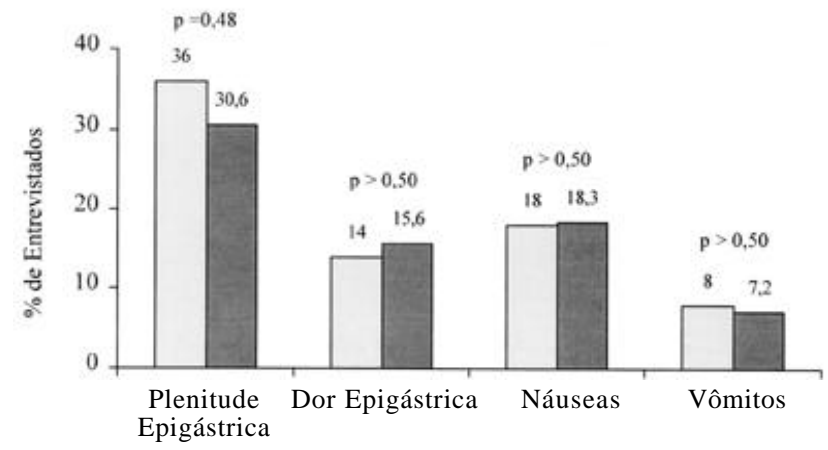

Controles $\square$ Diabéticos

\section{Discussão}

Os resultados do presente estudo indicam que pacientes brasileiros com Diabetes Mellitus apresentam prevalência elevada de sintomas digestivos. No entanto, entre os controles, a freqüência de sintomas digestivo também foi alta e, na comparação entre os dois grupos, quanto à freqüência de sintomas específicos, somente a de disfagia foi significativamente superior nos diabéticos.

Um achado interessante foi a freqüência relativamente alta de manifestações digestivas nas pessoas aparentemente sadias que constituíram o grupo controle. De fato, 
como mostra a Figura I, 36\% dos controles apresentaram pelo menos uma queixa digestiva e mais de um terço destas pessoas referiram queixas específicas, como plenitude epigástrica (36\%) ou pirose (34\%). Estes dados são comparáveis aos de outros países, como, por exemplo, a Inglaterra, onde um estudo populacional recente revelou prevalência anual de 29\% para sintomas de refluxo gastro-esofagiano e de $25 \%$ para sintomas dispépticos $^{18}$. No Brasil, um inquérito sobre a freqüência de oito sintomas digestivos, incluindo pirose, dispepsia, dor abdominal, constipação e diarréia, em amostra de 279 estudantes de medicina, revelou prevalência variando de 20\% a 27\%, dependendo do sintoma considerado ${ }^{19}$.

Estes valores são semelhantes aos encontrados em estudos de prevalência de manifestações da síndrome de cólon irritável (dor ou desconforto abdominal, alteração abdominal, alteração do hábito intestinal, distensão abdominal, sintomas defecatórios) feitos em outros países, como a de $22 \%$ na Inglaterra ${ }^{20}$ e de $15 \%$ na Noruega ${ }^{21}$ e de $17 \%$ nos Estados Unidos da América ${ }^{25}$ e a de $20 \%$ na França 22 .

Tomados em conjunto, estes dados expressam, possivelmente, a alta prevalência real, na população geral, de condições como a doença do refluxo gastroesofageano, a úlcera péptica e, sobretudo dos distúrbios digestivos funcionais, como a dispepsia funcional e a síndrome do cólon irritável, nos quais os sintomas ocorrem na ausência de alterações orgânicas demonstráveis.

No grupo de diabéticos, a freqüência de muitos dos sintomas foi comparável à verificada em estudos feitos em outros países, como revela a Tabela 4. Nota-se, nesta tabela, a grande variação nas proporções observadas de um estudo para outro. Esta variação é, provavelmente, devida às diferenças nas características dos grupos estudados, com respeito à idade e à composição

Tabela 3 - Freqüência de alguns sintomas digestivos em subgrupos de pacientes (DM) diabéticos dos tipos I (DM I) e 2 (DM 2). Os dados apresentados são os números e, entre parênteses, as porcentagens de sujeitos com sintomas. Apresentam-se, também, os valores de p para a análise estatística (teste de Fisher) das diferenças entre os grupos.

\begin{tabular}{|c|c|c|c|}
\hline \multirow[b]{2}{*}{ Sintomas } & \multicolumn{3}{|c|}{ GRUPOS } \\
\hline & $D M I\left(n^{*}=26\right)$ & DM $2(n=127)$ & $p$ \\
\hline $\begin{array}{l}\text { - Pirose } \\
\text { - Disfagia } \\
\text { - Plenitude epigástrica } \\
\text { - Constipação Intestinal } \\
\text { - Diarréia } \\
\text { - Incontinência fecal }\end{array}$ & $\begin{array}{c}5(19,2) \\
1(3,8) \\
8(30,8) \\
5(19,2) \\
2(7,6) \\
3(11,5)\end{array}$ & $\begin{array}{c}40(31,5) \\
19(15,0) \\
38(29,9) \\
21(16,5) \\
9(7,0) \\
4(3,1)\end{array}$ & $\begin{array}{l}>0,50 \\
>0,50 \\
>0,50 \\
>0,50 \\
>0,50 \\
>0,50\end{array}$ \\
\hline${ }^{*} \mathrm{n}$ - número de part & & & \\
\hline
\end{tabular}

Figura 4 - Porcentagens de pacientes dos grupos de diabéticos (coluna hachadura, $n=153$ ) e de voluntários do grupo controle (coluna clara, $n=50$ ) apresentando diferentes sintomasintestinais. Não houve diferenças significativas entre os dois grupos quanto à frequêencia de qualquer destes sintomas.

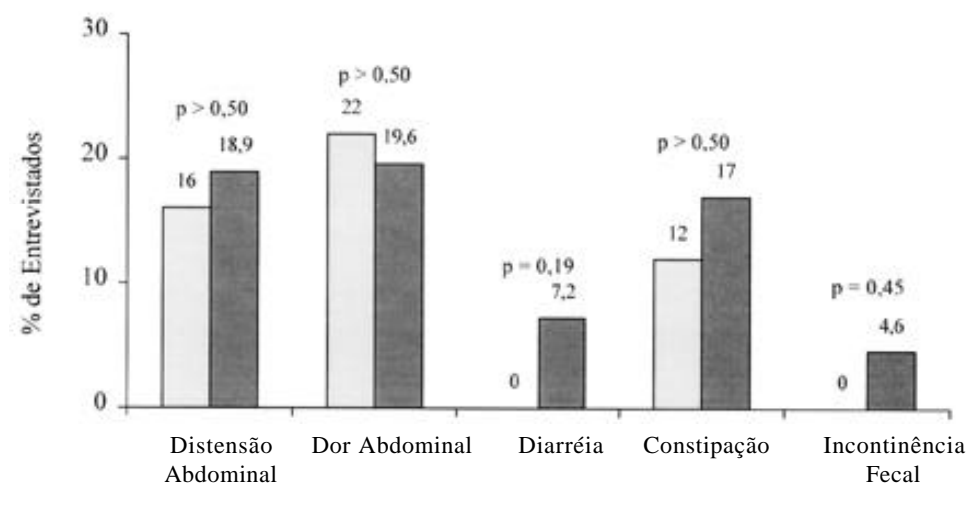

Controles

Diabéticos

por sexo, bem como às proporções relativas dos dois tipos clínicos do diabetes. No presente estudo, as freqüências verificadas entre os diabéticos situaram-se dentro, ou próximas à faixa de variação dos trabalhos citados, mas registraram-se proporções relativamente baixas de disfagia (13\%) e de diarréia (7\%).

É importante considerar, porém, que a comparação estatística das freqüências de sintomas encontrados em diabéticos somente foi efetuada em poucos estudos ${ }^{5-11}$. No estudo de Maxton \& Whorwell ${ }^{9}$, de
1991, apenas o sintoma constipação intestinal foi significativamente mais prevalente em diabéticos do que em controles. Já no estudo de Janatuinen et al. ${ }^{10}$, de 1993, não houve qualquer diferença entre $48 \mathrm{I}$ diabéticos e 635 controles quanto à prevalência de qualquer sintoma gastrintestinal. Em outro estudo realizado na Alemanha" não houve quaisquer diferenças entre diabéticos do tipo I e controles quanto à freqüência de sintomas digestivos variados e verificou-se prevalência superior à dos controles, no grupo de diabéticos do tipo 2, so- 
TRONCON LEA ET AL.

Tabela 4 - Freqüência comparativa da ocorrência de alguns sintomas digestivos em relatos de outros países e no presente estudo.

\begin{tabular}{|c|c|c|c|c|c|c|c|c|c|}
\hline \multirow{2}{*}{$\begin{array}{l}\text { Autor } \\
\text { principal }\end{array}$} & \multirow{2}{*}{$\begin{array}{c}\text { Ref. } \\
\mathrm{n}^{0}\end{array}$} & \multirow[t]{2}{*}{ Ano } & \multirow[t]{2}{*}{ País } & \multirow[t]{2}{*}{$\mathrm{n}^{*}$} & \multicolumn{5}{|c|}{$\%$ de casos referindo sintomas } \\
\hline & & & & & Pirose & Disfagia & $\begin{array}{c}\text { Plenitude } \\
\text { epigástrica }\end{array}$ & Constipação & Diarréia \\
\hline Feldman & 2 & 1983 & USA & 136 & - & 27 & 27 & 60 & 22 \\
\hline Clouse & 8 & 1988 & USA & 114 & - & - & - & 12 & 20 \\
\hline Maxton & 9 & |99| & UK & 200 & - & - & - & 16 & 8 \\
\hline Janatuinen & 10 & 1993 & Fin & 481 & 44 & 13 & - & 41 & 42 \\
\hline Troncon & - & & $\mathrm{BR}$ & 153 & 30 & 13 & 30 & 17 & 7 \\
\hline
\end{tabular}

*n - número de pacientes; USA - Estados Unidos da América; UK - Reino Unido; Fin - Finlândia; BR - Brasil.

mente quanto à constipação intestinal.

Este conjunto de observações confirma que pacientes portadores do Diabetes Mellitus apresentam alta prevalência de sintomas gastrointestinais. No entanto, permanece incerto se a freqüência de cada manifestação clínica, individualmente, é significativamente superior à da população geral, de modo a garantir relevância clínica específica a cada um destes sintomas. Esta interpretação implica em questionar a associação entre sintomas digestivos e as alterações fisiopatológicas trazidas pelas complicações gastrintestinais do diabetes, tão bem demonstradas em inúmeros trabalhos ${ }^{2-7,23-25}$.

A dissociação entre sintomas e alterações motoras no diabetes tem sido comprovada, por outro lado, em estudos que demonstram alta freqüência de refluxo gastro-esofageano ${ }^{26}$ ou de retarde do esvaziamento gástrico ${ }^{24}$ em diabéticos completamente assintomáticos. Nestes casos, a ausência de sintomas pode ser explicada pelo comprometimento das vias neurais aferentes, como parte do quadro de neuropatia diabética ${ }^{27}$, do que pode resultar diminuição considerável da sensibilidade visceral. Este mesmo fenômeno parece ser análogo ao que explica a maior prevalência de isquemia miocárdica silenciosa em pacientes diabéticos ${ }^{28}$.

Por outro lado, nos diabéticos com sin- tomas digestivos, a origem destas manifestações pode estar ligadas não só às alterações fisiopatológicas do tubo digestivo, mas também à ocorrência de outros fatores envolvidos na gênese dos distúrbios funcionais não-orgânicos, como a síndrome do cólon irritável ou a dispepsia funcional. De fato, em um estudo em diabéticos norte-americanos $^{8}$ verificou-se que os sintomas sugestivos das doenças do tubo digestivo proximal, bem como as alterações do hábito intestinal, estiveram mais significativamente associados com transtornos psiquiátricos do que com a presença de neuropatia periférica. Sugeriram os autores que as queixas ligadas ao trato gastrointestinal em diabéticos possam corresponder àquelas síndromes funcionais não-orgânicas tão comuns na população geral ${ }^{8}$. De fato, a condição de portador de uma doença crônica pode ser fator importante na produção de sintomas digestivos não associados a comprometimento orgânico do trato gastrointestinal ${ }^{29}$.

Do ponto de vista prático, os resultados deste trabalho sugerem que a detecção, na história clínica de pacientes diabéticos de sintomas digestivos, como os aqui investigados, não implica, necessariamente, na ocorrência de complicações gastrointestinais do diabetes. Estes sintomas podem ser devidos a doenças digestivas comuns, entre as quais os distúrbios funcionais, como a dispepsia funcional e a síndrome do cólon irritável. Escapa desta noção o sintoma disfagia, cuja prevalência foi significativamente maior entre os diabéticos que nos controles.

Com efeito, a ocorrência de disfagia em diabéticos parece indicar, com maior grau de segurança, a existência de distúrbios da peristalse esofagiana ${ }^{30,31}$, que, por sua vez, decorre de comprometimento da inervação autonômica do esôfago ${ }^{3 !}$. Não é possível descartar, porém, que a diferença estatística observada entre as proporções de pacientes e controles com disfagia tenha ocorrido por acaso, o que tem a probabilidade de acontecer muito aumentada em situações que exigem comparações múltiplas, como no presente trabalho. No entanto, é interessante notar que isso tenha eventualmente ocorrido exatamente na análise das prevalências de disfagia e não com relação a outros sintomas menos específicos. A disfagia, no diagnóstico diferencial entre afecções orgânicas e funcionais do tubo digestivo, é considerada como uma queixa sugestiva de comprometimento orgânico do esôfago ou da junção esofagogástrica $^{32}$

Por outro lado, mesmo considerando que a maior prevalência de disfagia nos diabéticos seja verdadeira, não é implausível que este sintoma decorra de outras causas de distúrbios da motilidade esofágica, como por exemplo a doença de Chagas ou a 
neuropatia alcóolica ${ }^{33}$, cuja ocorrência não foi determinada neste estudo.

Outras possíveis exceções ao entendimento de que a presença de sintomas digestivos pode não decorrer, necessariamente, de complicações do diabetes sobre motilidade digestiva relacionam-se às queixas de diarréia e incontinência fecal, que não foram registradas em qualquer dos controles. É plausível que, no caso destes dois sintomas, o estudo de um número maior de casos pudesse trazer significância estatística às diferenças encontradas entre diabéticos e controles, do que resultaria maior significado para a detecção destes dois sintomas.

\section{Conclusóes}

Em conclusão, os dados deste estudo mostram que a prevalência de sintomas digestivos em diabéticos brasileiros é relativamente alta, atingindo até $30 \%$ dos pacientes para alguns sintomas, como a pirose e a plenitude gástrica. No entanto, a comparação com uma amostra de pessoas sadias revelou que somente a queixa de disfagia foi significativamente mais comum nos diabéticos. É possível, portanto, que a freqüência elevada de sintomas digestivos em diabéticos decorra mais de condições comuns, como as síndromes funcionais digestivas não-orgânicas, do que de complicações crônicas do diabetes. As freqüências encontradas das diferentes queixas estiveram próximas das relatadas em estudos feitos no hemisfério Norte, o que não apóia a hipótese de que fatores externos ao meio possam influenciar a ocorrência de sintomas digestivos em diabéticos.

\section{SUMMARY}

\section{Digestive SYMPTOMS IN BRAZILIAN patients with Diabetes Mellitus}

PuRPose. To determine the frequencies of digestive symptoms in an unse- lected sample of Brazilian diabetics, in comparison to those verified in the general population.

MEtHods. The frequencies of 13 digestive symptoms were determined in 153 type 1 and type 2 diabetics and in 50 apparently healthy contro ls, utilizing a structured, standardized questionnaire.

Results. The percentage of diabetics with at least one symptom was significantly higher than in controls (70\% vs $36 \%, p=$ 0.01 ). Higher frequencies of upper digestive symptoms, such as postprandial epigastric fullness (30\% vs 35\%), heartburn (30\% vs $34 \%$ ), as well as constipation (I $7 \%$ vs 12\%) were observed in both groups. Nevertheless, only the prevalence of dysphagia ( $13 \%$ vs $2 \%$ ) was significantly increased ( $p=$ $0.02)$ in diabetics.

Conclusion. These findings indicate that gastrointestinal symptoms are common in diabetics, but this seems also to be the case in the general population, with the exception of dysphagia. The frequencies of symptoms observed in Brazil were similar to those reported in studies from the North Hemisphere, a finding that does not support the hypothesis that external factors may influence the prevalence of gastrointestinal symptoms in diabetics. [Rev Ass Med Brasil 2001; 47(2): |57-64]

KEYwORDS: Gastrointestinal symptoms. Diabetes mellitus. Dysphagia. Dyspepsia. Bowel habits. Functional disorders.

\section{Agradecimento}

Agradecemos ao Prof. Dr. Afonso Diniz Costa Passos, do Departamento de Medicina Social da FMRP-USP, pela orientação na análise estatística dos dados da versão final do manuscrito.

\section{REFERÊNCIAS}

I. Unger RH, Foster DW. Diabetes Mellitus. In: JD Watson, DW Foster, editors William's Texbook of Endocrinology $8^{\text {th }}$ ed., Philadel- phia, WB Saunders 1992: | 255- 1333.

2. Feldman M, Schiller LR. Disorders of gastrointestinal motility associated with diabetes mellitus. Ann Intern Med 1983; 98: 378-384.

3. Rosa-e-Silva L, Troncon LEA, Oliveira RB, Braga FJHN, Gallo jr. L. Foss MC. Rapid distal small bowel transit associated with sympathetic denervation in type I diabetes mellitus. Gut 1996; 39: 748-756.

4. Falchuk KR, Conlin D. The intestinal and liver complications of diabetes mellitus. Adv Intern Med 1993; 38: 269-285.

5. Rosa-e-Silva L. Troncon LEA, Oliveria RB, lazigi N, Gallo Jr. L., Foss MC. Treatment of diabetic gastroparesis with oral clonidine. Alimentary Pharmacology \& Therapeutics 1995; 9: 179- | 83.

6. Kassander P. Asymptomatic gastric retention in diabetes (gastroparesis diabeticorum). Ann Inter Med 1958; 48: 797-8।2.

7. Katz LA, Spiro HM. Gastrintestinal manifestations of diabetes. N Engl J Med 1986; 275: |350- |36|.

8. Clouse ME, Lustman PJ. Gastrointestinal symptoms in diabetic patients. Lack of association with neuropathy. Am J Gastroenterol 1988; 84: 868-872.

9. Maxton DG, Whorwell PJ. Functional bowel symptoms in diabetes - the role of autonomic neuropathy. Postgrad Med J 1991; 67: 991-993.

I0. Janatuinen E, Pikkarainen P, Laakso M, Pyorala K. Gastrointestinal symptoms in middle-aged diabetic patients. Scand J Gastroenterol 1993 ; 28: 427-432.

II. Enck P, Rathmann W, Spiekermann M, Czerner d, Tschope D, Ziegler D, Strohmeyer G, Gries FA. Prevalence of gastrotintestinal symptoms in diabetic patients and non-diabetic subjects. Z Gastroenterol 1994: 32: 637-64l.

12. Schwarcz E, Palmer M, Ingberg CM, Aman J, Berne $C$. Increased prevalence of upper gastrointestinal symptoms in long-term type I diabetes mellitus. Diabet Med 1996; 13: 478-481.

13. National Diabetes Data Group. Classification and diagnosis of diabetes mellitus and other categories of glucose intolerance. Diabetes 1979: 28: 1039- 1057.

14. World Medical Association declaration of Helsinki. Recommendations guiding physicians in biomedical research. JAMA 1997; 277: 925-926.

15. Drossman DA. Bowel patterns among subjects not seeking health care. Gastroenterology 1982; 83: 529-534.

16. Talley NJ, Boyce P, Jones M. Identification of distinct upper and lower gastrointestinal symptom groupings in an urban population. Gut 1998; 42: 690-695.

17. Siegel S. Non-parametric satistics for the behavioural sciences. McGraw-Hill, 1956.

18. Kennedy TM, Jones RH, Hungin AP, O'Flanagon $\mathrm{H}$, Kelly $\mathrm{P}$. Irritable bowel syndrome, gastro-oesophageal reflux and bronchial hyper-responsiveness in the general population. Gut 1998; 43: 770-774.

19. Sobral DT, Vidigal KS, Farias-e-Silva K. Diges- 
tive symptoms in young individuals: survey among medical students. Arq Gastroenterol 1991; 28: 27-32.

20. Jones R, Lydeard S. Irritable bowel syndrome in the general population. Brit Med J 1992; 304: 87-90.

21 . Agreus $L$. The epidemiology of functional gastrointestinal disorders. Eur J Surg (Suppl) 1998; 583: 60-66.

22. Bonmelier G, Rouch M, Dapoigny M, Pais D, Loisly P, Gualino M, Tournut R. Epidemiology of intestinal functional disorders in an apparently healthy population. Gastroenterol Clin Biol 1986; 10: 7-12.

23. Rothstein RD. Gastrointestinal motility disorders in diabetes mellitus. Am J Gastroenterol 1990; 85: 782-785.

24. Horowitz M, Wishart JM, Jones KL, Hebbard GS. Gastric emptying in diabetes: an overview. Diabet Med 1996; 13 (suppl 5): 516-522.
25. Camilleri M. Gastrointestinal problems in diabetes. Endocrinol Metab Clin North Am 1996; 25: 361-378.

26. Lluch I, Ascaso JF, Mora F, Minguez M, Pena A, Hernandez A, Benages A. Gastroesophageal reflux in diabetes mellitus. Am J Gastroenterol 94: 919-924, 1999.

27. Ewing DJ, Clarke BF. Diagnosis and management of diabetic autonomic neuropathy. Br Med J 1982; 285: 916-918.

28. Naka M, Hiramatsu K, Aizawa T, Momose A, Yoshizawa K, Shigematsu S, Ishihara F, Niwa A, Yamada T. Silent myocardial ischemia in patients with non-insulin-dependent diabetes mellitus as judged by treadmill exercise testing and coronary angiography. Am Heart J 1992; 123: 46-53.

29. Drossman DA. Psychosocial factors in gastrointestinal disorders. In: M Feldman, BF Schar Schmidt, MH Sleisenger, editors Sleisenger \& Fordtran's Gastrointestinal and Liver Disease, $6^{\text {th }}$ ed., Philadelphia, WB Saunders Co 1998; 69-79.

30. Hollis JB, Castell DO, Braddon RL. Esophageal function in diabetes mellitus and its relation to peripheral neuropathy. Gastroenterology 1977; 73: 1098-11 02.

31. Souza MAN. Caracterização da motilidade esofagiana e do esvaziamento gástrico em pacientes diabéticos com e sem refluxo esofagiano. Tese de Doutorado, Faculdade de Medicina de Ribeirão Preto da Universidade de São Paulo, 1998.

32. Miller RM. Clinical examination for dysphagia. In: ME Groher, editor, Dysphagia, $3^{\text {rd }}$ ed., Boston, Butterworth-Heineman 1997; 169-190.

33. Castro LP. Esôfago - Distúrbios da Motilidade. In: R. Dani, LP Castro, editores. Gastroenterologia Clínica, $3^{a}$ edição, Rio de Janeiro, Guanabara-Koogan, 1993; $36|-37|$.

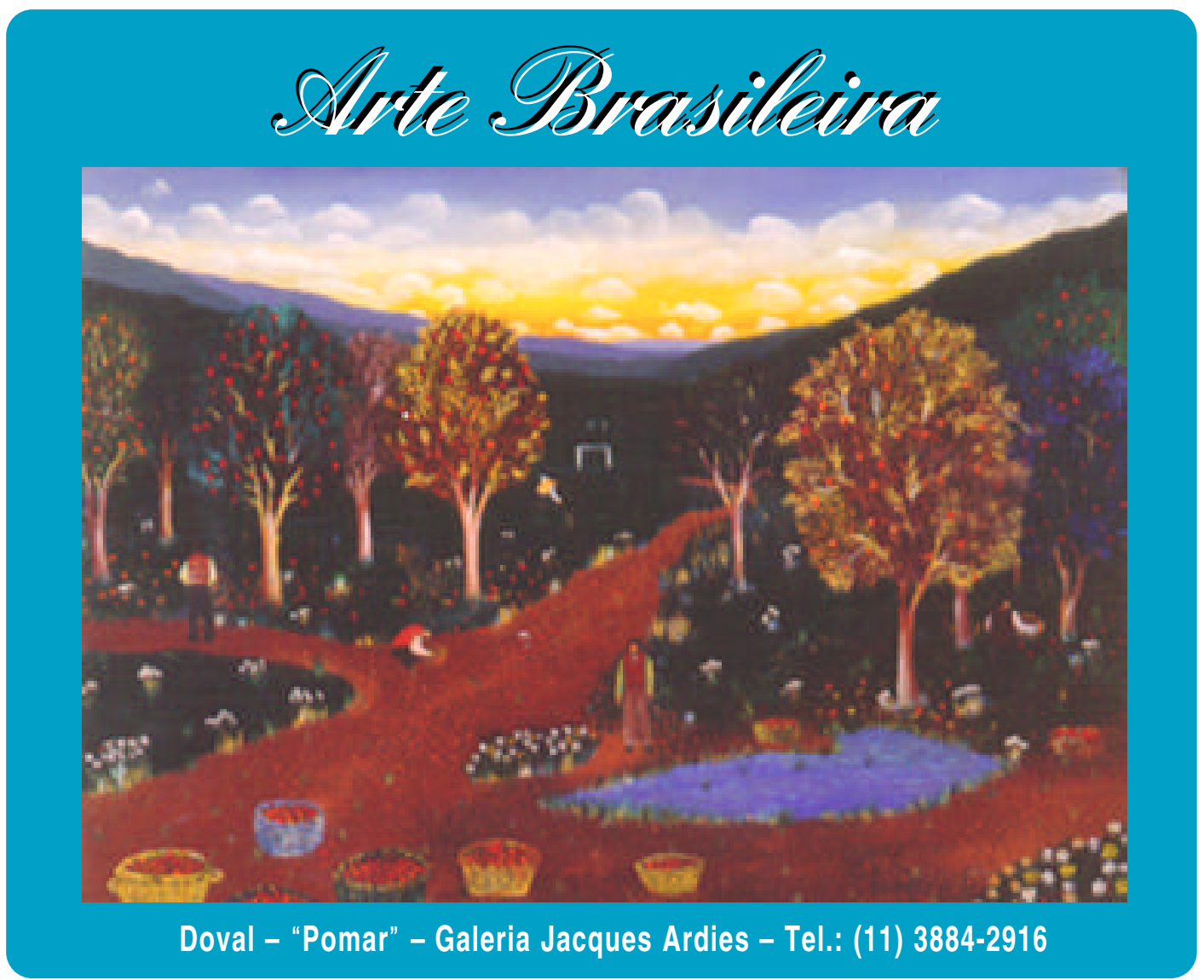

